\title{
Vers une naturalisation de la filiation ?
}

Agnès Fine, Agnès Martial

Genèses, vol. 78, no. 1, 2010, pp. 121-134.

La filiation peut-elle être l'objet d'une interrogation sur la «biologisation» ou la «rebiologisation » des questions sociales ? C'est la question qui guidera la présente contribution, abordée sous l'angle de l'anthropologie des relations de parenté contemporaines.

Une telle interrogation semble légitime au vu des débats politiques et juridiques récents. Choisissons, parmi d'autres, deux exemples. Durant l'automne 2007, de vives polémiques ont accompagné le vote d'un amendement au projet de loi Hortefeux sur l'immigration, qui instaurait la possibilité du recours aux tests ADN dans les procédures de regroupement familial, les candidats pouvant apporter la preuve de leur filiation par un test génétique. Soulignant l'incompatibilité d'un tel projet avec le contenu de la filiation en droit français et les règles bioéthiques qui prévalent à l'utilisation des tests $\mathrm{ADN}$, elles dénonçaient la réduction des liens parents-enfants à une stricte dimension génétique et la stigmatisation des familles étrangères qui s'y trouveraient alors soumises, la loi créant pour elles une catégorie spécifique de filiation. Elles soulignaient également, pour démontrer l'inadaptation d'une telle conception des liens parents-enfants à la société française contemporaine, l'importance des parentés «sociales » incarnées notamment par les recompositions familiales. Ainsi en août 2007, la lettre de mission du président de la République au ministre concerné, évoquait-elle en ces termes le projet d'une reconnaissance légale du rôle des beaux-parents dans les familles recomposées :

\footnotetext{
« Vous créerez un statut du beau-parent dont l'objectif est de permettre aux adultes qui vivent avec des enfants dont ils ne sont pas les parents biologiques de pouvoir procéder pour eux aux démarches habituelles de la vie quotidienne, et de protéger juridiquement, dans l'intérêt de tous, les liens affectifs incontestables qui se nouent entre ces enfants et ces adultes. » ${ }^{1}$
}

Cette formulation visait sans doute à décrire simplement la situation des beaux-parents élevant dans les familles recomposées des enfants qu'ils n'ont effectivement pas conçus. Mais en distinguant parents et beaux-parents en fonction d'une relation «biologique » à l'enfant,

http://www.elysee.fr/elysee/elysee.fr/francais/interventions/2007/juillet/lettre de mission_adr essee a $m$ xavier bertrand ministre du travail des relations sociales et de la solidarite.7 9212.html, consulté le 15 novembre 2008. 
elle peut mener, une fois déplacée dans la sphère politique et juridique, à la confrontation de deux formes de relations parentales. Les débats tenus sur le forum de l'association SOS Papa en sont une illustration : exprimant leur inquiétude quant aux possibles effets de la reconnaissance d'un statut beau-parental sur la fragilisation des relations père-enfant, certains participants y opposent, en référence à la lettre de mission gouvernementale, le «vrai » parent non gardien, parfois décrit comme le «parent naturel » ou le «père génétique » au parent social incarné par le beau-père de l'enfant ${ }^{2}$. Pourtant, la relation biologique ne produit en ellemême ni droits ni devoirs tant qu'elle n'est pas traduite en terme de filiation paternelle, que celle-ci soit instaurée par le mariage, par la reconnaissance ou par l'adoption.

Ces exemples, relevant de débats et d'objectifs politiques séparés (les conditions d'entrées des migrants sur le territoire français dans un cas, le projet de répondre aux attentes éventuelles des beaux-parents dans les familles recomposées dans l'autre), interrogent néanmoins tous deux les conceptions de la parenté en révélant comment une relation de droit, la filiation, peut être confondue avec une relation résultant de la procréation, le lien génétique ou biologique. Cette assimilation d'un lien à l'autre traverse de manière plus générale nombre de débats sur les nouvelles formes de vie familiale, où l'on tend bien souvent à opposer une parenté « sociale » à une parenté «biologique », en oubliant, pour le dire simplement, que la seconde n'est pas moins « sociale » que la première.

Ces interprétations sont à replacer dans le contexte des changements familiaux contemporains, qui ont conduit à la mise en doute des certitudes sur lesquelles s'appuyait la définition de la filiation. Le «démariage» (Théry 1993, 2007) et la suppression des distinctions entre filiations dites « légitimes » et «naturelles » ${ }^{3}$ ont profondément bouleversé l'univers juridique de l'alliance et de la filiation. Alors que plus de $50 \%$ des enfants naissent hors mariage en France ${ }^{4}$, l'institution du mariage n'est plus le fondement exclusif du lien conjugal et des relations parents-enfants. L'augmentation et la banalisation des trajectoires conjugales impliquant ruptures et recompositions familiales ont eu pour effet de dissocier

2. http://www.sospapa.net/phpBB2/viewtopic.php?p=50323

Nous remercions Anne Verjus d'avoir porté ce débat à notre connaissance.

3. Ordonnance portant réforme de la filiation, 4 juillet 2005 , entrée en vigueur le $1^{\text {er }}$ juillet 2006.

4. INED, « Population en chiffres » :

http://www.ined.fr/fr/pop_chiffres/france/naissances_fecondite/naissances_hors_mariage/, consulté le 5 février 2010. 
conjugalité et filiation, tout en ajoutant beaux-parents, demi et quasi-frères et sœurs à l'univers familial de nombre d'enfants. Les familles adoptives témoignent d'une séparation entre sexualité, procréation et filiation, comme d'autres configurations nouvelles. Les nouvelles technologies biomédicales ont ainsi permis que soient fécondés des embryons hors du corps de la mère, dans le cas des fécondations in vitro, puis de convoquer autour de la conception d'un enfant des donneurs de gamètes masculins ou féminins, et enfin des «mères porteuses » dans le cadre de la gestation pour autrui, la maternité devenant un processus fragmenté où sont investis plusieurs corps de femmes. Des couples de même sexe ont recours aux nouvelles techniques de reproduction ou à l'adoption et accèdent dans certains pays à la reconnaissance légale des liens qui les unissent à leurs enfants, contribuant encore à dissocier la procréation de la filiation et ouvrant une nouvelle ère où la différence des sexes ne serait plus constitutive de la fondation d'une famille.

La profusion et la richesse des travaux portant depuis trente ans sur les redéfinitions contemporaines de la parenté euro-américaine témoignent de l'importance des interrogations suscitées par ces changements. Nombre d'entre eux ont adopté, pour analyser la complexité de ces nouvelles formes de parenté, une distinction conventionnelle visant à analyser les significations attribuées à la dimension «sociale » ou «biologique » de la filiation dans les représentations culturelles des liens entre parents. Comme on l'a vu, ces catégories analytiques ont parfois été reprises et simplifiées dans le débat public, réduisant la filiation à l'une ou l'autre de ces dimensions. Cette distinction est également rigidifiée par certaines analyses proposant une alternative entre deux formes de parenté, dont l'une serait sociale tandis que la seconde, sous l'effet des progrès des sciences médicales et/ou de leur interprétation juridique, mènerait à la «biologisation » de la filiation. Un récent exemple de telles interprétations est exposé dans l'ouvrage de Marcela Iacub, L'empire du ventre (2004). Dans son analyse de la traduction juridique de la maternité en droit français, elle fait l'hypothèse d'une «naturalisation» de la filiation maternelle, illustrée par la valeur grandissante accordée à la gestation et à la mise au monde. Pour elle, en France, la prohibition de la gestation pour autrui serait l'expression d'une sacralisation du ventre maternel interdisant la possibilité qu'existe une maternité fondée sur la volonté. Ouvrant la focale à $\mathrm{d}$ 'autres situations familiales singulières, elle les interprète dans une logique identique. La non reconnaissance d'une double filiation des enfants nés et élevés dans des couples de même sexe serait liée à leur non adéquation avec un modèle procréatif hétérosexuel dominant le droit de la filiation. Dans le contexte de l'adoption, les revendications des mouvements de lutte pour la reconnaissance des origines émanant des enfants adoptés ou nés sous X seraient 
l'expression d'un attachement inconditionnel à l'appartenance «biologique » des individus. La valorisation de la dimension naturelle de l'engendrement impliquerait finalement une conception de la filiation arbitraire et productrice d'inégalités : entre les hommes et les femmes d'une part, ces dernières étant ainsi ramenées à leur statut de mères et de reproductrices, entre les parents fertiles et ceux qui ne peuvent concevoir naturellement, d'autre part. Une alternative serait alors de fonder la filiation sur la seule volonté des individus. Daniel Borillo (2009), à partir d'exemples différents, conclut lui aussi à une naturalisation croissante de la filiation.

Le modèle à partir duquel s'est juridiquement construite la filiation en droit français est en effet le couple hétérosexuel, et la reconnaissance légale d'une double filiation dans les familles homoparentales rencontre encore bien des obstacles ${ }^{5}$. On peut cependant discuter le caractère «procréatif » de ce modèle, que d'autres auteurs situent plutôt dans l'histoire de l'institution du mariage (Théry 2007). Il est vrai que l'évolution juridique récente a accentué en Europe le caractère «naturel » de la paternité par le recours au test génétique en cas de conflit de filiation (Martial 2008 ; Salazar 2009) et de la maternité. En France, depuis le 4 juillet 2005, l'inscription du nom de la mère sur l'acte de naissance de l'enfant « vaut reconnaissance » : celle-ci n'a désormais plus besoin d'aller reconnaître son enfant, le seul fait biologique de l'accouchement étant retenu pour justifier l'existence juridique du lien maternel. Cependant, l'analyse menée par Marcela Iacub et Daniel Borillo, enclose dans l'étude de l'histoire du droit et vouée à la défense d'un nouveau modèle juridique de filiation, ne tient aucun compte des représentations et des usages de la filiation dans l'espace occidental. Son hypothèse présume que cette filiation puisse être réduite à la dimension «naturelle» de l'engendrement, ce qui paraît fort discutable du point de vue de l'anthropologie de la parenté. L'argumentation opposant une parenté uniquement fondée sur la «nature » à une autre, appuyée sur la volonté et la dimension sociale des liens, témoigne en outre d'une grande méconnaissance de l'univers des relations entre parents dans le monde occidental, qu'ont éclairé nombre de travaux d'ethnologie et de sociologie. Pour l'expliciter, nous exposerons ici les principales avancées théoriques et empiriques des recherches d'histoire et d'anthropologie menées sur la parenté euro-américaine depuis près de quarante ans. Un premier rappel de l'histoire même de ces recherches est nécessaire.

5. Pour une revue de la reconnaissance juridique de l'homoparentalité dans les divers pays européens, voir Waaldijk 2009. 


\section{De l'ordre symbolique de la «nature " à l'étude des parentés «parallèles » dans l'histoire de l'anthropologie de la parenté en Europe}

Pour qui s'intéresse à la parenté dans les sociétés occidentales contemporaines, les analyses de David Schneider (1968) concernant les représentations symboliques de la parenté euroaméricaine, forgées à partir d'une étude menée au sein de familles des classes moyennes de Chicago à la fin des années 1960, sont incontournables, et placent au centre de la parenté euro-américaine la question des faits de «nature ». Carles Salazar en a récemment synthétisé les apports avec une grande clarté. D'après Schneider, écrit-il, « une relation de parenté est une catégorie produite par la combinaison de deux ordres symboliques distincts : l'ordre de la nature et celui de la loi. Selon le premier, les parents sont ceux qui partagent des substances naturelles - des substances biogénétiques - symbolisées dans les discours par le terme "sang". Selon le second, les parents sont ceux qui respectent un certain code de conduite. » (Salazar 2005 : 31). Or, les relations basées sur le «sang» ont plus de valeur que celles qui sont uniquement basées sur la loi, car elles sont indéfectibles, quel que soit le code de conduite adopté. Elles sont en outre composées de deux formes d'amour entre parents : l'amour conjugal et l'amour parental, le cœur symbolique de l'ensemble du système reposant sur l'acte procréatif, c'est-à-dire sur les rapports sexuels donnant lieu à la naissance d'un enfant, lié ensuite à ses parents par le partage d'un même matériel biogénétique (Salazar 2005). Le constat de l'importance accordée par les sociétés occidentales à la dimension naturelle et biogénétique des liens entre parents mènera David Schneider à contester la pertinence des études de parenté menées dans d'autres cultures par les anthropologues anglo-américains, conduits selon lui à projeter sur des sociétés différentes leur propre système de significations et de symboles dès lors qu'ils prétendent que «la parenté, les généalogies et les liens biologiques sont universels » (Salazar 2005 : 38 ; Schneider 1984, Viveiros de Castro 2004). Ses travaux vont par ailleurs influencer la plupart des recherches menées sur la parenté en Europe et en Amérique du Nord, au moment où se développent de nouvelles configurations relationnelles interrogeant la cohérence et la hiérarchie établie par Schneider entre l'ordre de la «nature » et l'ordre de la «loi »: les familles adoptives, les familles recomposées, et les familles constituées grâce à l'assistance médicale à la procréation. Du fait même de leur composition, ces modèles familiaux suggèrent une possible distinction entre une composante «biologique » et une composante «sociale» des relations. Un ensemble de recherches se

développent alors sur les parentés dites «parallèles» ou encore «fictives» ou 
« artificielles », termes traditionnellement adoptés par les anthropologues pour désigner des relations volontaires créées sur le modèle de certains liens de sang. Interrogeant la valorisation de la dimension «naturelle » des liens entre parents, ces relations sont analysées dans le temps de l'histoire comme dans l'étude du contemporain. Commençons par l'histoire.

\section{Idéologie du sang et parentés parallèles dans l'Europe ancienne}

Depuis l'Antiquité, les cultures occidentales attribuent une importance non négligeable aux liens dits «naturels ».

\footnotetext{
« Nombreuses sont, dans le monde grec, les parentés dites "fictives", en ce sens qu'elles proviennent de conventions et non de liens biologiques. Il n'en reste pas moins que fondamentalement, et étymologiquement, la parenté en grec, donc la sungeneia, est définie par l'origine commune, et repose sur la reconnaissance de liens considérés par les Grecs eux-mêmes comme naturels » (Wilgaux 2005 : 77).
}

La culture grecque valorise la notion d'hérédité à travers la transmission des traits physiques, des qualités personnelles et des comportements, fondant certaines distinctions sociales sur la détention d'un «sang pur » qu'il faut protéger des mésalliances.

Cette valorisation du sang comme vecteur d'identité et d'appartenance est également bien connue pour le Moyen Âge. À partir du $\mathrm{x}^{\mathrm{e}}$ siècle, l'essor du lignage accompagne la féodalisation, témoignant de «l'enracinement de l'idéologie du sang dans la culture médiévale » (Bresc 1981 : 394). La pratique des généalogies est un riche reflet de l'imaginaire médiéval de la parenté : Christiane Klapish-Zuber analyse notamment le recours à la figure de l'arbre comme représentation des lignées, le lien végétal portant parfois, « comme une sève, le filet de sang qui circule entre les générations et les soude en une communauté » (2000:323). Les ricordanze florentins reconstituent ainsi des généalogies familiales fondées sur le sang et le nom d'où les femmes sont les grandes absentes, exclues des lignages en raison de leur incapacité à hériter. Si l'amour maternel est alors déjà valorisé comme naturel (Carron 1989), il s'ancre dans la gestation et la mise au monde plus que dans le sang partagé. Car c'est en donnant la vie aux enfants du lignage que les femmes y prennent place.

La valorisation des liens de sang semble si forte dans l'Europe chrétienne qu'elle paraît éclipser toute autre forme de parenté. L'adoption, pratiquée en Grèce antique (Leduc 1998) comme à Rome (Moreau 1992 ; Corbier 1999) disparaît ainsi des règles du droit de la fin de l'Empire romain jusqu'à la Révolution française (Gutton 1993). Elle ne sera véritablement 
rétablie qu'avec le Code civil napoléonien, et réservée aux majeurs, l'adoption des mineurs ne datant en Europe que des années 1920. Cette disparition demeure un mystère encore irrésolu (Fine 1999), mais que l'influence conjuguée de la féodalité et de l'Église permet d'éclairer du point de vue des conceptions juridiques et culturelles de la parenté. Faut-il en conclure que le sang partagé devient à cette époque la seule forme de relation concevable entre parents et enfants, l'unique support métaphorique des représentations de la parenté ? Ce serait oublier d'autres dimensions possibles des relations entre personnes.

Dans le monde chrétien, le lien créé par le baptême en est la plus claire illustration (Fine 1994). Le baptême est pensé comme «une seconde naissance, une naissance spirituelle nécessitant de nouveaux parents différents des géniteurs, parce que ces derniers sont souillés par l'acte de reproduction » associé par la doctrine chrétienne au péché originel (Fine 1998b: 90). Lors du baptême, les parrains et marraines sont censés «refaire l'enfant », à travers la transmission d'un prénom, d'une identité sexuée, et de certaines de leurs qualités morales ou physiques. Le parrainage crée une relation toujours positive qui s'oppose à l'ambivalence de la filiation par le sang. La parenté baptismale unit également parrains et parents d'un même enfant dans une relation de compérage, une amitié sacrée marquée par des termes d'adresses spécifiques, par l'usage du vouvoiement et caractérisée par un interdit sexuel entre parents de sang et parents spirituels, interdit qui existe aussi entre parrains et filleuls. La parenté spirituelle donne ainsi lieu à un don d'enfant symbolique qui inaugure un ensemble riche et complexe d'échanges et de relations. Elle déjoue de ce fait « la parenté charnelle, qui porte en elle les risques de l'inceste et du repliement sur soi » (Fine 1994 : 99). En distinguant parenté spirituelle et parenté charnelle, l'Église «naturalise» la seconde qui met en jeu la reproduction sexuée et confère à la première une valeur sacrée, garantissant ainsi son rôle social. Un rôle social d'une efficacité remarquable puisqu'elle est créatrice de liens indéfectibles aussi bien dans le monde des vivants que dans l'au-delà. L'existence de cette relation tout au long de l'histoire chrétienne suggère la pluralité des manières de penser la parenté dans l'Europe ancienne.

Bien que marginalisée, l'adoption continue par ailleurs d'exister, revêtant des formes discrètes, éparses, occultées. On en trouve quelques traces à l'époque médiévale (Lett 2000). On peut en rechercher l'empreinte, sous l'Ancien Régime, au gré des placements des nombreux enfants orphelins ou abandonnés dans les hospices, au sein de familles où ils représentent une force de travail fort utile, les parents de naissance ou l'hospice conservant cependant la patria postestas exercée sur les enfants (Klapisch-Zuber 1999 ; Delille 1999). La possibilité de transmettre un héritage par voie testamentaire est par ailleurs demeurée ouverte 
depuis le Moyen Âge (Delille 1999). Elle peut donner lieu à des «adoptions testamentaires », étudiées par Kristin Gager (1999) chez les artisans et commerçants parisiens et par Roger Aubenas (1934) chez les propriétaires ou artisans méridionaux de la fin du Moyen Âge. Dans ces cas, «l'adoption se fait devant notaire, par un contrat entre adoptant et parents de l'adopté organisant une donation entre vifs ou par testament» (Fine 1998b: 64). Réalisées par un couple ou une personne célibataire sans descendance, ces adoptions tendent d'abord à pallier le manque d'héritier afin d'assurer la continuité d'une lignée en voie d'extinction. Elles permettent aussi à l'adoptant sans conjoint ni descendance de former avec l'adopté une communauté «de travail et de résidence qui lui permettra d'affronter la vieillesse avec un soutien ", et le rassurent quant à l'accomplissement des rites et des gestes indispensables, après son décès, à la survie de son âme dans l'autre monde. Dans le Sud-Ouest des années 1930 aux années 1960, des adoptions de fait ou des adoptions simples légales se font en milieu rural, même modeste, avec les mêmes objectifs. Elles ont lieu, en général, au sein de la parenté, dans «une culture parfaitement hostile à l'intégration familiale d'un étranger au sang », sur lequel pèsent divers soupçons de bâtardise, d'inceste, et de tares héréditaires (Fine $1998 b$ : 69). «La solution préférée est d'adopter au plus près, c'est-à-dire quelqu'un de son propre sang », le choix se tournant de manière préférentielle vers le neveu ou la nièce (ibid. : 70). Justifié par des motifs de solidarité et des stratégies patrimoniales en accord avec l'idéologie de la lignée, un tel don peut en outre passer inaperçu dès lors que l'enfant est donné à la naissance à un frère ou une sœur. On observe cependant, au tournant des années 1950, l'évolution des représentations entourant ces pratiques qui reflète le changement profond des conceptions de la parenté et de la place de l'enfant dans la famille (Ariès 1973). À partir des années 1950, «le don volontaire d'un enfant par sa mère devient asocial, véritablement contre nature » (Fine 1998b:85). Assimilé à une vente, il devient impossible, impensable. La norme de l'amour maternel, dont la puissance s'accroît au fil du XIX ${ }^{\mathrm{e}}$ siècle et durant la première moitié du Xx ${ }^{\mathrm{e}}$ (Badinter 1980 ; Knibiehler et Fouquet 1980), est venue transformer le don d'enfant en abandon (Fine 1998b). La valorisation du «sang » comme métaphore de l'unité du lignage liant les générations se serait ainsi déplacée vers la relation mère-enfant.

Concluons ce rappel historique : la référence à la dimension «biologique » des relations de parenté, portée par la métaphore du sang partagé, traverse de part en part l'histoire culturelle des sociétés d'Europe occidentale. Elle n'est cependant jamais l'unique mode de construction de la parenté et coexiste de manière indissociable - puisque tous les enfants sont baptisés avec la parenté spirituelle, très investie et valorisée dans le monde chrétien. Elle 
s'accompagne aussi, à partir du Moyen Âge, de formes discrètes d'adoption qui doivent s'accommoder de l'idéologie lignagère jusqu'à ce que celle-ci s'efface au profit de la valorisation des relations mère-enfant, ce qui transformera profondément, nous le verrons, le sens même des relations adoptives.

Même si elle est pensée et vécue en référence à une parenté spirituelle posée comme idéale, la référence à la «dimension biologique » de la filiation n'a donc rien de nouveau dans nos sociétés. À l'aune de la longue durée historique, outre la disparition de la croyance à l'audelà, le changement contemporain le plus important ne se situe donc pas dans une «biologisation » des conceptions de la parenté mais tout au contraire dans le développement, la reconnaissance juridique et la valorisation de liens parents-enfants non soutenus par le sang et la chair partagés. Ces relations nouvelles ont été étudiées par la sociologie et l'anthropologie à partir de différentes configurations : les familles recomposées, les familles adoptives, et les familles constituées grâce à l'assistance médicale à la procréation. Comme on va le voir, ces différents modèles constituent de féconds révélateurs de la diversité des fondements des liens entre parents, mais aussi de la redéfinition constante de la référence au «biologique ».

\section{Nouvelles parentés parallèles}

\section{Dans les familles recomposées}

Aux parâtres et marâtres de l'Europe ancienne, incarnation d'une parenté malfaisante usurpant la place d'un parent, père ou mère, décédé, ont succédé de nouvelles figures parentales, étudiées à travers l'émergence de relations informelles, plurielles, dont le caractère inédit, par comparaison avec les familles issues des remariages après veuvage du passé, était d'ajouter un beau-père et/ou une belle-mère aux père et mère séparés de l'enfant (Théry et Meulders-Klein 1993 ; Cadolle 2000).

Agnès Martial $(2003,2005)$ a mis en évidence l'importance du partage de l'enfance et de l'éducation dans la manière dont se construisent des relations profondément électives entre un enfant et l'adulte, ou les adultes, qui l'ont élevé sans pour autant l'avoir désiré et conçu. De même, des formes de fraternité nouvelle, inscrites dans la corésidence et le partage d'une histoire familiale commune ont été mises au jour (Martial 1998 ; Poittevin 2006). L'étude du traitement de la notion d'inceste, menée à travers les représentations et l'interprétation 
d'éventuels interdits sexuels entre ces différents « apparentés », ainsi que dans l'analyse de la pratique judiciaire associée aux cas de violences sexuelles commises par un beau-parent sur la personne de son bel-enfant, a permis de formuler une définition de «l'inceste beau-parental» conditionnée à la réalité sociale et affective : le beau-parent coupable de violence envers l'enfant de son conjoint était condamné avec la circonstance aggravante de «personne ayant autorité » (équivalente à celle qui frappait un ascendant de l'enfant) si - et seulement si - il s'était comporté auparavant « comme un parent » envers cet enfant (Martial 2003).

En outre, de nouvelles pratiques successorales témoignent de la volonté de faire circuler les biens par d'autres voies que la seule filiation reconnue juridiquement, dès lors qu'un beaupère ou une belle-mère souhaite transmettre ses biens à l'enfant de son conjoint. L'adoption simple alors utilisée ne supprime pas la parenté d'origine de l'enfant mais instaure une filiation additionnelle. Les beaux-parents, sauf cas particulier, ne remplacent pas le parent «biologique» mais coexistent avec lui. Creusets d'une possible pluriparenté, les familles recomposées tendent donc à relativiser l'importance des relations consanguines au fondement de la filiation. Ceci n'exclut pas cependant le recours à la métaphore du sang partagé dans la justification des liens de familles qui s'y développent, la demi-fraternité étant particulièrement investie dès lors que naît un enfant du couple parent-beau-parent.

Dans des cas rares et particuliers, concernant des enfants nés sans filiation paternelle et reconnus par le conjoint de la mère, la dimension «biologique » des relations peut aussi réapparaître dans le contexte d'un conflit judiciaire portant sur les liens paternels de l'enfant. L'analyse d'une trentaine de dossiers judicaires retraçant, après la séparation du couple, des contestations de filiation paternelle a révélé le caractère arbitraire de certaines ruptures de filiation (Martial 2008). Une expertise génétique ou hématologique est en effet demandée et considérée comme une «preuve absolue de la filiation » (Pousson-Petit 2002 : 431), qui n'est plus suspendue qu'à la réalité du sang partagé. Dans quelques dossiers cependant, le refus de renoncer à leur paternité mène certains hommes à déployer de riches argumentations sur la valeur sociale, matérielle et affective de leur rôle auprès de l'enfant, évitant parfois le couperet de l'expertise. On voit ainsi se dessiner l'inextricable entrelacs des références au sang, au nom, et la nécessaire actualisation de la filiation dans les interactions affectives, économiques et matérielles de la parenté «pratique » qui se déploient entre adultes et enfants dans les familles dissociées et recomposées (Weber 2005).

\section{Dans la parenté adoptive}


L'adoption connaît dès la seconde moitié du XIX ${ }^{\mathrm{e}}$ siècle aux États-Unis (Carp 1998) et à partir de l'entre-deux-guerres dans la partie occidentale de l'Europe un important développement, allié à une profonde redéfinition de son contenu. Les anthropologues l'ont largement démontré (Modell 1993; Fine 1998a; Fine et Neirinck 2000; Ouellette 1998, 2000) : elle crée aujourd'hui une relation filiale à part entière. L'adoption s'est par ailleurs développée bien audelà des frontières nationales, et s'est trouvée médiatisée et valorisée comme un geste généreux et altruiste à l'égard d'enfants orphelins ou abandonnés issus de pays pauvres. De ce fait aussi, la majorité des enfants adoptés aujourd'hui ne peuvent plus ressembler à leurs parents : une nouvelle image de la filiation adoptive, devenue l'incarnation de relations fondées sur le choix et la volonté, s'est progressivement construite. Les études portant sur l'adoption notent cependant la place fantasmée que continue à occuper la mère de naissance, parfois évoquée par les mères adoptives elles-mêmes comme la «vraie mère » de l'enfant, dans l'univers familial. Ainsi, dans l'adoption, des liens nouveaux se construisent sans que la référence aux liens de sang s'efface tout à fait, comme en témoignent les revendications liées à la connaissance des origines, portées en France et aux États-Unis par certains enfants adoptés et nés sous X, ou les enquêtes menées en Grande-Bretagne par Janet Carsten (2004) qui mettent en évidence le sentiment d'incomplétude ressenti par des enfants par ailleurs bien intégrés dans leur famille adoptive. Les débats suscités par la question de l'accès à la connaissance des origines et plus largement l'étude des mécanismes institutionnels et juridiques de l'adoption ont mis en évidence, dans nos sociétés, la très grande réticence des systèmes juridiques et des représentations culturelles à reconnaître l'existence, autour d'un individu, de plus d'un seul père et d'une seule mère (Ouellette 1998 ; Fine 1998a; Fine et Neirinck 2000). Un modèle exclusif de filiation préside en effet à la traduction juridique de la filiation adoptive. Il rejoint celui de l'engendrement biologique, qui n'implique que deux individus de sexe différent dans la conception de l'enfant, et s'allie à notre système de parenté cognatique, qui en reconnaissant à Ego deux groupes de parents, paternel et maternel, semble reproduire les conditions de l'engendrement. Dans la filiation adoptive, le droit efface la relation issue de la conception de l'enfant, pour la remplacer par une relation sociale qui s'efforcera de paraître aussi « vraie » qu'un lien biologique. Ceci s'accompagne bien souvent du secret porté sur les origines de l'enfant, l'adoption plénière (votée en France en 1966) rompant les liens de l'enfant à son ou ses parents de naissance, afin de donner aux parents adoptifs le statut plein et entier de père et de mère : un nouvel acte de naissance est rédigé, comme si l'enfant était véritablement «né» de ses nouveaux parents. Ceci permet en outre 
d'éviter toute interconnaissance entre les donneurs et les preneurs d'enfants, et de contourner ainsi les réticences morales liées au don d'enfant dans nos sociétés (Ouellette 2004).

Les travaux menés par Françoise Romaine Ouellette et Caroline Méthot au Québec montrent que la norme du secret tend à disparaître au profit d'un « consensus relatif pour la constitution d'une mémoire des origines de l'enfant et une volonté de l'intégrer à la dynamique familiale » (2003 : 143). Ce mouvement est soutenu par la mise en place de divers services encadrant les recherches des adoptés internationaux dans de nombreux pays et association. En l'absence d'informations précises sur ses origines personnelles, «les efforts de constitution d'une mémoire pour l'enfant se font en référence à son pays et à sa culture d'origine plutôt qu'en référence à des personnes et à sa famille de naissance. Cette mémoire s'amarre surtout aux souvenirs de voyage des parents adoptifs et à leurs connaissances livresques. Elle transpose alors la problématique des origines en dehors du champ de la parenté. À travers ce détour, l'opposition entre filiation biologique et adoptive se dissout» (ibid.). Ce qui se joue dans l'attachement de ces enfants à une culture dont ils sont devenus étrangers est moins leur origine au sens «biologique » que la construction, pour chacun d'eux, d'une histoire fondant leur identité personnelle.

\section{Dans les nouvelles technologies de reproduction}

Le recours à l'assistance médicale à la procréation peut être analysé comme l'expression d'une préférence pour la composante «biologique » de la filiation. Les statistiques relatives au choix des différente techniques médicales d'assistance à la procréation montrent ainsi le succès de la fécondation in vitro, les réticences à recourir à l'insémination avec donneur en cas d'échecs, et l'utilisation croissante de l'ICSI (Intracytoplasmic Sperm Injection) qui permet d'assurer la fécondation d'un ovule avec le sperme du père de l'enfant, même dans les cas d'infertilité masculine sévères, en dépit des risques encourus pour la santé de la mère et des inquiétudes concernant le développement ultérieur des enfants (Rochebrochard 2003). Une dernière possibilité est le recours à la gestation pour autrui, existant déjà sous une forme légalisée dans de nombreux pays ${ }^{6}$. On peut présumer que la motivation première des couples infertiles ayant recours à la gestation pour autrui serait d'avoir un enfant qui soit

6. Pour une revue de l'encadrement juridique très variable selon les pays, voir « La gestation pour autrui ", Les documents de travail du Sénat, Série législation comparée, $\mathrm{n}^{\circ}$ LC 182, janvier 2008, http://www.senat.fr/lc/lc182/lc182.htm (consulté le15 novembre 2008). 
génétiquement relié à l'un au moins des deux parents - le père - (Ragoné 1996), parfois aux deux parents lorsque la mère d'intention est aussi la mère génétique de l'enfant. Ces nouvelles techniques de procréation traduisent donc un fort investissement de l'engendrement biologique, qui s'exerce concrètement, du point de vue médical, sur le corps des femmes (Tain 2001). La progression des savoirs et des techniques rend donc possible un désir qui semble toujours plus explicitement affirmé par les couples infertiles, soutenus par l'encadrement médical : avoir un enfant de sa chair, de son sang, fondant ainsi le lien filial dans la « biologie ».

Il semble cependant que la réalité soit autrement plus complexe. Tout d'abord, les nouvelles techniques de reproduction sont également productrices de formes inédites de parenté « sociale ». Dans le cas des inséminations avec donneur, c'est le conjoint de la mère qui est désigné comme le père juridique, s'engageant à élever comme le sien l'enfant issu des gamètes d'un autre homme (Delaisi 1998). La gestation pour autrui et le don d'ovocyte instaurent en revanche une paternité «certaine » au sens génétique, quand la maternité peut faire l'objet d'une dissociation de ses composantes biologique et sociale, distinguant la mère génétique, la mère porteuse et la mère sociale (Tain 2004 ; Delaisi et Collard 2007). La gestation pour autrui interroge ainsi la norme qui fonde la maternité dans la gestation et la mise au monde. Héléna Ragoné montrait comment les femmes américaines engagées dans un tel processus distinguaient et valorisaient une maternité nourricière comme instance première de la création du lien mère-enfant, ce dernier étant conçu «dans le cœur » de la mère d'intention («conception in the heart »), tandis que la dimension biologique du processus de maternité se trouvait dévalorisée (Ragonné 1996). Mais ces observations sont aujourd'hui nuancées par l'étude des pratiques récentes qui évoquent l'idée d'une grossesse partagée par les deux femmes, la mère d'intention étant très fortement impliquée dans la gestation. En outre, la pratique de l'allaitement de l'enfant par les mères d'intention met en évidence le fait «que la maternité corporelle n'est pas définie de façon exclusivement anténatale » et se rejoue finalement entre la mère d'intention et l'enfant après la naissance (Delaisi et Collard $2007: 41)$.

Comment, dans ce contexte, distinguer les dimensions «sociale» ou «biologique » de la filiation? La plupart des auteurs qui ont étudié le recours à l'assistance médicale à la procréation contestent la prévalence du «tout génétique»(Gardey 2006 ; Franklin et Ragonné 1998). Les interventions technologiques dans la reproduction sont toujours réinterprétées pour que se fabriquent - ou non - des liens d'ascendance. Elles produisent de nouvelles façons de tracer les distinctions entre parents et renforcent en même temps des 
manières plus anciennes de construire les identités (Thompson 2001 ; Bestard 2005). Les informations génétiques sont « appropriées », « incorporées » par les individus, produisant les corps, les relations et les identités (Franklin 2001 ; Strathern 1992). On peut alors interroger la notion même de «biologie » ou de «nature » telle qu'elle est invoquée pour expliquer l'existence des liens entre parents et enfants, en distinguant «la pensée concrète de la parenté à la pensée scientifique de la science » (Bestard 2005 : 141). Les supports de connaissance et de représentation des «faits de nature » ont évolué. On observe un «changement de polarité dans les représentations de la parenté : celle-ci s'est déplacée de la consubstantialité vers les gènes »(Delaisi et Collard $2007: 41$ ), ce qui implique un partage qui concerne bien plus l'information sur les qualités de la personne que la «substance » ou le sang partagé (Carsten 2007). C'est le cas dans le contexte de la gestation pour autrui, lorsque la mère d'intention est aussi la mère génétique. Le discours sur les gènes apparaît comme la manière moderne de parler des ressemblances physiques entre parents et enfants, comme il ressort des nombreuses recherches sur les nouvelles techniques de procréation (Fortier 2009). Les CECOS (Centres d'Études et de Conservation des Oeufs et du Sperme) prennent soin d'apparier au mieux géniteurs et parents d'intention. De manière plus étonnante, on découvre que certains organismes d'adoption font de même lorsqu'ils attribuent un enfant à des parents adoptifs (Bestard et Marre 2004). Quant aux enfants qui ignorent leur ascendance de naissance, loin de contester leur filiation adoptive à laquelle ils sont généralement très attachés, ils disent tout simplement vouloir savoir «à qui ils ressemblent». C'est pour cette raison et surtout pour comprendre les raisons de leur abandon que les enfants adoptés revendiquent la connaissance de leur histoire. Cette quête n'est évidemment pas réductible à une valorisation quelconque du «biologique », d'autant qu'elle réfère également au champ de l'appartenance ethnique, culturelle ou nationale. Au fil de ces déplacements, l'enjeu demeure toujours le même. La métaphore du biologique ou des gènes, la référence à la culture ou à la nation renvoient à la problématique des origines qui permettent de parachever la construction de récits capable de singulariser les enfants tout en les intégrant dans un univers de parenté donné. En effet, comme le rappellent nombre d'auteurs s'inspirant des thèses de Paul Ricœur, «répondre à la question “qui ?”, c'est raconter une histoire » (Hannah Arendt, citée par Théry 2007 : 601 ; Ouellette et Saint-Pierre 2008).

En conclusion, nous pouvons désormais apporter quelques éléments de réponse à la question posée dans le titre de cet article. On ne peut, du point de vue de l'anthropologie de la parenté, 
considérer que la filiation fait aujourd'hui l'objet d'une «biologisation » ou d'une «naturalisation» particulièrement nouvelle. La référence aux circonstances naturelles de la procréation, dans les discours sur la parenté, a été et demeure une puissante métaphore, car le corps est dans nos cultures «un moyen privilégié »d'entrer en filiation (Delaisi et Collard 2007 : 41). Elle n'en coexiste pas moins avec la composante sociale des liens entre parents, d'autant plus visible aujourd'hui que ces différentes facettes sont dissociées. La contextualisation des interprétations et des pratiques associées aux relations entre parents est alors essentielle, afin de ne pas survaloriser le pouvoir de la «biologie ». La richesse des études anthropologiques est justement, à partir d'observations minutieuses, toujours replacées dans un contexte historique et social singulier, d'identifier les changements qui affectent les représentations de la «nature » dans la parenté.

Il semble donc ne pas exister de relation qui se passe tout à fait d'une référence aux circonstances naturelles de la mise au monde, pas plus qu'il n'existe de lien «biologique » suffisant à lui seul à fonder une relation parent-enfant. Dissocier et opposer ces deux dimensions n'a pas de sens du point de vue des représentations de la parenté, car elles participent ensemble de la construction des univers familiaux contemporains comme elles participaient de celle des univers anciens. En fait, « le problème survient quand le biologique et le social sont distingués et qu'il faut donner sens à l'un d'entre eux seulement » (Bestard 2005 : 145). La difficulté est de faire exister conjointement ces deux dimensions, dès lors qu'elles s'incarnent dans une possible pluralité de parents. Dans son introduction à un livre collectif intitulé Parents de sang, parents adoptifs (2000), Agnès Fine a rapproché des configurations que l'on peut définir comme pluriparentales et que l'on peut observer dans le domaine de l'adoption, en particulier aux USA et au Québec, de celle des procréations médicalement assistées et des familles recomposées ${ }^{7}$. On pourrait aujourd'hui ajouter à cet ensemble les familles homoparentales. L'enjeu actuel est de reconnaître chacune de ces parentalités dans leur spécificité, juridique et sociale. Discuter ces questions implique de clarifier les notions utilisées, en replaçant l'usage des références à la «nature » ou à la « biologie » dans la pluralité des registres interprétatifs de la parenté européenne.

\section{Ouvrages cités}

7. Voir également Fine 2001. 
ARIES, Philippe. 1973 [1960]. L'enfant et la vie familiale sous l'Ancien Régime. Paris, Seuil (L'Univers historique), ( $1^{\text {re }}$ éd., Paris, Plon).

Aubenas, Roger. 1934. L'adoption en Provence au Moyen Âge, XIV ${ }^{e}-X V I^{e}$ siècle. Paris, Librairie du Recueil Sirey.

BADINTER, Élisabeth. 1980. L'amour en plus. Histoire de l'amour maternel, XVII'-XX ${ }^{e}$ siècle. Paris, Flammarion.

BestaRd, Joan. 2005. «Parenté, ethnicité, race et nation : sur les différents modes de partage de substances biogénétiques », Incidences, $\mathrm{n}^{\circ}$ 1, «Qu'est-ce que la parenté ? »: 131-148.

— et Diana MARRE. 2004. «El cuerpo familiar; personas, cuerpos y semejanzas », in J. Bestard et D. Marre (éd.), La adopción y el acogimiento. Presente y perspectivas. Barcelone, Universitat de Barcelona (Estudis d'antropologia social i cultural) : 293-312.

BoRILLO, Daniel. 2009. «La vérité biologique contre l'homoparentalité : le statut du beauparent ou le "PACS de la filiation" ", Droit et sociétés, vol. 2, n 72 : 259-271.

BRESC Henry, 1981, «L'Europe des villes et des campagnes (XIII ${ }^{\circ}-\mathrm{XV}^{\circ}$ siècle) », BURGUIERE A., Klapisch C., Segalen M., Zonabend F. (Dir.), Histoire de la famille, Paris, Armand Colin T.1, pp. 385-419.

CADOlle, Sylvie. 2000. Etre parent, être beau-parent. La recomposition de la famille. Paris, Odile Jacob.

CARP, E. Wayne. 1998. Family Matters. Secrecy and Disclosure in the History of Adoption. Cambridge (Mass.), Harvard Univesity Press.

CARron, Roland. 1989. Enfant et parenté dans la France médiévale, $X^{e}$-XIII siècles. Genève, Droz (Travaux d'histoire éthico-politique).

CARsten, Janet. 2004. After Kinship. Cambridge, Cambridge University Press. 
- 2007. «Constitutive Knowledge : Tracing Trajectories of Information in New Contexts of Relatedness», Anthropological Quarterly, vol. 80, n 2 : 403-426.

CORBIER, Mireille. 1999. «La petite enfance à Rome : lois, normes, pratiques individuelles et collectives », Annales. Histoire, sciences sociales, n 6 : 1257-1290.

DELAISI, Geneviève. 1998 [1881]. La part du père. Seuil.

— et Chantal COLLARD. 2007. «La gestation pour autrui. Un bricolage des représentations de la parenté et de la maternité euro-américaines », L'Homme, $\mathrm{n}^{\circ} 183$ : 29-54.

DELILLE, Gérard. 1999. «La non adoption : orphelinats et Monts de mariage en Italie (XVII ${ }^{\mathrm{e}}$ XVIII ${ }^{\mathrm{e}}$ siècles) », in Mireille Corbier (éd.), Adoption et fosterage. Paris, De Boccard (De l'archéologie à l'histoire) : 357-368.

FINE, Agnès. 1994. Parrains, marraines. La parenté spirituelle en Europe. Paris, Fayard.

- (éd.). 1998a. Adoptions. Ethnologie des parentés choisies. Paris, MSH (Ethnologie de France).

— 1998b. «Le don d'enfants dans l'ancienne France », in A. Fine (éd.) : 61-96.

- 1999. «Adoption et parrainage dans l'Europe ancienne », in Mireille Corbier (éd.), Adoption et fosterage. Paris, De Boccard (De l'archéologie à l'histoire) : 357-368.

— 2001. «Vers une reconnaissance de la pluriparentalité ? », Esprit, n 273, «L'un et l'autre sexe $»: 40-52$.

FINE, Agnès et Claire NEIRINCK (éd.). 2000. Parents de sang, parents adoptifs. Approches juridiques et anthropologiques de l'adoption. Paris, LGDJ (Droit et société).

FORTIER, Corinne. 2009. «Quand la ressemblance fait la parenté », in Enric Porqueres i Gené (éd.), Défis contemporains de la parenté. Paris, EHESS (Cas de figure) : 251-276. 
FRANKLIN, Sarah. 2001. «Biologisation Revisited : Kinship Theory in the Context of the New Biologies », in Sarah Franklin et Susan McKinnon (éd.), Relatives Values. Reconfiguring Kinship Studies. Durham, Duke University Press : 302-325.

- et Héléna Ragonné (éd.). 1998. Reproducing Reproduction: Kinship, Power and Technological Innovation. Philadelphia, University of Pennsylvania Press.

GaGeR, Kristin. 1999. «Adoption Practices in Sexteenth and Seventeeth Century Paris », in Mireille Corbier (éd.), Adoption et fosterage. Paris, De Boccard (De L'Archéologie à l'histoire) : 183-198.

GARDEY, Delphine. 2006. «Les sciences et la construction des identités sexuées. Une revue critique », Annales. Histoire, sciences sociales, $\mathrm{n}^{\circ} 3$ : 649-673.

Gutton, Jean-Pierre. 1993. Histoire de l'adoption. Paris, Publisud (Courants universels. Histoire).

IACUB, Marcela. 2004. L'empire du ventre. Pour une autre histoire de la maternité. Paris. Fayard (Histoire de la pensée).

KLAPISCH-ZubeR, Christiane. 1999. «L'adoption impossible dans l'Italie de la fin du Moyen Âge », in Mireille Corbier (éd.), Adoption et fosterage. Paris, De Boccard (De L'Archéologie à l'histoire) : 321-338.

— 2000. L'ombre des ancêtres. Essai sur l'imaginaire médiéval de la parenté. Paris, Fayard (L'Esprit de la cité).

KNIBIEHLER, Yvonne et Catherine FOUQUET. 1980. L'histoire des mères du Moyen Âge à nos jours. Paris, Montalba.

LEDUC, Claudine. 1998. «L'adoption dans la cité des Athéniens à l'époque classique », in Agnès Fine (éd.), Adoptions. Ethnologie des parentés choisies. Paris, MSH (Ethnologie de la France) : 45-60. 
LETT, Didier. 2000. Famille et parenté dans l'Occident médiéval, $V^{e}-X V^{e}$ siècle. Paris, Hachette (Carré histoire).

MARTial, Agnès. 1998. «Partages et fraternité dans les familles recomposées », in A. Fine (éd.) : 206-244.

- 2003. S'apparenter. Ethnologie des liens de familles recomposées. Paris, MSH (Ethnologie de la France).

— 2005, «Comment rester liés ? Les comptes des familles recomposées », Terrain, $\mathrm{n}^{\circ} 45$ : $67-82$.

— 2008. «Changements de nom, changement de filiation», in Agnès Fine (éd.), États civils en questions. Papiers, identités, sentiment de soi. Paris, CTHS (Le Regard de l'ethnologue) : 115-138.

ModELl, Judith S. 1993. Kinship with Stranger. Adoptions and Interpretations of Kinship in American Culture. Berkeley, University of California Press.

MOREAU, Philippe. 1992. «Les adoptions romaines », Droit et cultures $n^{\circ} 23$, « Adoption et transferts d'enfants » : 13-30.

OuELLETTE, Françoise Romaine. 1998. «Les usages contemporains de l'adoption », in A. Fine (éd.) : 153-176.

— 2000. «L'adoption face aux définitions de la famille et de l'institution généalogique », in A. Fine et C. Neirinck (éd.) : 325-342.

— 2004. «Adopter, c'est donner », in Isabelle Leblic (éd.), De l'adoption. Des pratiques de filiation différentes. Clermont-Ferrand, Presses universitaires Blaise Pascal (Anthropologie) : 269-296.

— et Caroline Meнот. 2003. «Les références identitaires des enfants adoptés à l'étranger : entre rupture et continuité », Nouvelles Pratiques sociales, vol. 16, $\mathrm{n}^{\circ} 1$ : 132-147. 
Ouellette, Françoise Romaine et Julie SAINT-PIERRE. 2008. «La quête des origines en adoption internationale. Être chez soi et étranger », Informations sociales, ${ }^{\circ} 146$ : 84-91.

Poittevin, Aude. 2006. Enfants de familles recomposées. Sociologie des nouveaux liens fraternels. Rennes, Pur (Le sens social).

Pousson-Petit, Jacqueline (éd.). 2002. L'identité de la personne humaine. Étude de droit français et de droit comparé. Bruxelles, Bruyant.

RAGONÉ, Héléna. 1996. «Chasing the Blood Ties : Surrogate Mothers, Adoptive Mothers and Fathers », American ethnologist, vol. 23, n $2: 352-365$.

ROCHEBROCHARD (DE), Élise. 2003. «Des hommes médicalement assistés pour procréer : IAD, FIV, ICSI, bilan d'un révolution de la prise en charge médicale de l'infertilité masculine », Population, vol. 58, $\mathrm{n}^{\circ} 4-5$ : 549-586.

SALAZAR, Carles. 2005. « David M. Schneider et l'anthropologie de la parenté », Incidences, $\mathrm{n}^{\circ} 1$, «Qu'est-ce que la parenté ? » :25-49.

- 2009. «Vérité biologique et fiction sociale dans l'histoire du droit paternel. Essai d'anthropologie juridique », in Enric Porqueres i Gene (éd.), Défis contemporains de la parenté. Paris, EHESS (Cas de figure) : 59-80.

SCHNEIDER, David M. 1968. American Kinship : a Cultural Account. Englewood Cliffs (NJ), Prentice-Hall (Anthropology of Modern Societies series).

- 1984. A Critique of the Study of Kinship. Ann Arbor, University of Michigan Press.

STRATHERN, Marilyn. 1992. Reproducing the Future. Anthropology, Kinship and the New Reproductive Technologies. Manchester (UK), Manchester University Press.

TAIN, Laurence. 2001. «L'hôpital, la femme et le médecin. La construction de trajectoires de fécondation in vitro », Population, vol. 56, $\mathrm{n}^{\circ} 5: 811-844$. 
— 2004. «Genre et techniques de reproduction : évidences, alliances et turbulences », L’homme et la société, n $1562-1563: 229-246$.

THERY, Irène. 1993. Le démariage. Justice et vie privée. Paris, Odile Jacob.

— 2007. La distinction de sexe. Une nouvelle approche de l'égalité. Paris Odile Jacob.

— et Marie-Thérèse MEulders-KLeIN (éd.). 1993. Les recompositions familiales aujourd'hui. Paris, Nathan (Essais et recherches).

Thompson, Charis. 2001. «Strategic Naturalizing : Kinship in an Infertility Clinic (California) », in Sarah Franklin et Susan McKinnon (éd.), Relatives Values. Reconfiguring Kinship Studies. Durham, Duke University Press : 175-202.

VIVEIROS de CASTRO, 2004, "Le don et le donné : trois nano-essais sur la parenté et la magie", Ethnographiques.org, $\mathrm{n}^{\circ} 6$ (en ligne), http://www.ethnographiques.org/2004/Viveiros -de-Castro

WAALDIJK, Kees. 2009. « Overview of Forms of Joint Legal Parenting Available to Same-Sex Couples in European Countries », Droit et sociétés, $\mathrm{n}^{\circ} 72: 383-385$.

WEBER, Florence. 2005. Le sang, le nom, le quotidien. Une sociologie de la parenté pratique. La Courneuve, Aux Lieux d'être (Mondes contemporains).

WiLgauX, Jérome. 2005. «David Schneider en Attique : le sang, le sperme dans les représentations de la parenté en Grèce ancienne », Incidences, $\mathrm{n}^{\circ} 1$, «Qu'est-ce que la parenté ? » :131-148. 